\title{
PREVALÊNCIA DA LEISHMANIOSE VISCERAL CANINA E COINFECÇÕES EM REGIÃO PERIURBANA NO DISTRITO FEDERAL - BRASIL
}

\section{PREVALENCE OF VISCERAL CANINE LEISHMANIOSIS AND CO- INFECTIONS IN PERIURBAN REGION IN THE FEDERAL DISTRICT - $B R A Z I L$}

\author{
Cassio Ricardo Ribeiro 1 ORCID - http://orcid.org/0000-0003-3250-6674 \\ Carolynne Arruda Gonçalves² ORCID - http://orcid.org/0000-0002-0056-4442 \\ Lauricio Monteiro Cruz ${ }^{3}$ ORCID - http://orcid.org/0000-0001-5150-5867 \\ Paula Diniz Galera2* ORCID - http://orcid.org/0000-0002-6496-2538 \\ ${ }^{1}$ Médico Veterinário Autônomo, Brasília, DF, Brasil. \\ ¿Universidade de Brasília, Brasília, DF, Brasil. \\ ${ }^{3}$ Diretoria de Vigilância Ambiental em Saúde da Secretaria de Saúde do Distrito Federal, Brasília, DF, Brasil. \\ *Autora para contato - dra.paulagalera@gmail.com
}

\section{Resumo}

Objetivou-se verificar a prevalência da leishmaniose visceral canina (LVC) em área periurbana com foco de transmissão no Distrito Federal (DF) e também avaliar a prevalência de coinfecções como dirofilariose, anaplasmose, erliquiose e doença de Lyme em cães sororreagentes para LVC. Inicialmente, amostras de soros dos cães da área de estudo foram testadas à reação imunocromatográfica que contém dois antígenos recombinantes - rK39 e rK26 - (DPP) para Leishmania sp. O soro dos animais reagentes ao teste de DPP foi confirmado para LVC com ELISA. As amostras reagentes a ambos os testes foram analisadas para possíveis coinfecções através do teste de SNAP (IDEXX 4Dx Plus). Foram avaliados 240 cães, dos quais 132 (55\%) obtiveram resultados reagentes para DPP e para ELISA concomitantemente, sendo diagnosticados com LVC. Destes, 26 (20\%) foram reagentes para Ehrlichia canis ou E. ewangii, 9 (7\%) apresentaram positividade para Anaplasma phagocytophilum ou $A$. platys e, nessas amostras que se apresentaram reagentes, observou-se a ocorrência de coinfecção de anaplasmose, erliquiose e leishmaniose em 3 (2,3\%) animais. Verificou-se alta prevalência da LVC na região periurbana do $\mathrm{DF}$, destacando-se a necessidade de diagnóstico mais preciso quanto a coinfecções que possivelmente podem influenciar no prognóstico e manifestação do quadro clínico desses pacientes.

Palavras-chave: coinfecções, leishmania, DPP, ELISA

\begin{abstract}
The aim of this study was to verify the prevalence of Canine Visceral Leishmaniasis (CVL) in periurban area focusing on transmissions that occurred on Distrito Federal (DF), and also, evaluate the prevalence of co-infections like dirofilariosis, anaplasmosis, ehrlichiosis and Lyme disease in dogs seroreagents to CLV. Initially the serum of the dogs inhabitants of the study area was tested according to the immunochromatographic reaction that contained two recombinant antigens - rK39 e rK26 - (DPP) to Leishmania sp. The animal plasma that reacted to the DPP test was confirmed for CVL with ELI-SA. Through confirmation of diagnose of VL by both tests, the samples were analyzed
\end{abstract}


for possible co-infections through SNAP test (IDEXX 4Dx Plus). 240 dogs were tested, 132 (55\%) of which obtained reacting results for DPP and for ELISA concomitantly, being diagnosed with CVL. Amongst those, $26(20 \%)$ were positive for Ehrlichia canis or E. ewangii, meanwhile 9 (7\%) presented positive results for Anaplasma phagocytophilum or A. platys, from those samples that showed to be positive, co-infection of anaplasmosis, ehrlichiosis and leishmaniasis was observed in $3(2.3 \%)$ dogs. The high prevalence of CVL was observed in the periurban region of DF, highlighting the need for more precise diagnosis regarding co-infections that may possibly influence on the prognosis and clinical manifestation of these patients.

Key words: co-infections, Leishmania, DPP, Elisa

Recebido em: 02 de outubro de 2017.

Aceito em: 07 de junho de 2018.

\section{Introdução}

A leishmaniose visceral é uma antropozoonose cosmopolita potencialmente fatal causada por protozoários intracelulares obrigatórios pertencentes à família Trypanosomidae, gênero Leishmania $\operatorname{spp}^{(1,2)}$. No Brasil, a LV é causada pela Leishmania (Leishmania) chagasi - CUNHA \& CHAGAS, 1937-, tendo como principal vetor os flebotomíneos da espécie Lutzomyia longipalpis ${ }^{(2,3)}$, comumente conhecidos como mosquito-palha. Essa doença é a forma de apresentação mais severa dentre as leishmanioses, que classicamente são divididas em Leishmaniose Visceral (LV) e Leishmaniose Tegumentar (LT), sendo esta segunda forma de apresentação ocasionada, no Brasil, principalmente pelas espécies Leishmania amazonensis e L. brasiliensis ${ }^{(2)}$.

Para controlar a propagação de uma das doenças mais importantes da atualidade, principalmente em indivíduos imunossuprimidos, adotam-se medidas de controle preconizadas no Programa de Controle e Vigilância da Leishmaniose Visceral (PCVLV), que se baseia principalmente na eutanásia de cães sororreagentes, considerados os principais reservatórios urbanos do protozoário ${ }^{(2)}$. Isto porque têm íntima relação com o ser humano, bem como por serem altamente suscetíveis à infecção por Leishmania chagasi e representarem a principal fonte de infecção aos flebotomíneos por causa de parasitismo intenso na pele, independentemente da sua apresentação clínica ${ }^{(2,4,5,6)}$; no entanto, a eutanásia desses animais é uma medida controversa ${ }^{(8)}$.

Até 2012, a leishmaniose visceral canina (LVC) era diagnosticada utilizando-se a Reação de Imunofluorescência Indireta (RIFI), um método recomendado para a confirmação de casos positivos detectados por ELISA (Enzyme-Linked Immunosorbent Assay) ${ }^{(2)}$. Recentemente, para melhorar a acurácia no diagnóstico da LVC, o PCVLV recomenda a utilização de um teste rápido imunocromatográfico, compreendendo os antígenos recombinantes rK26 e rK39, o DPP (Dual-Path Plataform; Bio-Manguinhos / Fiocruz, Rio de Janeiro, Brasil), para a triagem de cães infectados, e ELISA para confirmar os resultados positivos $^{(8,9)}$.

A LV é uma doença endêmica principalmente em regiões tropicais e subtropicais e era registrada principalmente em áreas rurais ${ }^{(2,10)}$. Entretanto, nas últimas décadas, a doença vem se urbanizando à medida que o vetor tem se adaptado melhor a esses ambientes devido ao desequilíbrio ecológico, refletindo num aumento da incidência de casos. Verifica-se, ainda, que em poucos países a notificação é compulsória, e muitos casos não são devidamente diagnosticados, fazendo com que a incidência 
dessa doença seja subestimada ${ }^{(10)}$.

Nas últimas décadas, a LV tornou-se um importante problema de saúde pública no Brasil, afetando em torno de 3.400 pessoas por ano (estabilizado nesses valores anuais desde 2004), com uma taxa de incidência anual de 1,9 caso por 100.000 habitantes $^{(2)}$.

No Distrito Federal, os primeiros dois casos fatais e autóctones diagnosticados de LV humana foram observados no final de 2005, no Condomínio Serra Azul, Sobradinho II (área periurbana) ${ }^{(2)}$. Outros 65 casos humanos foram diagnosticados no Distrito Federal (2005-2013), em regiões periurbanas invadidas e ocupadas às custas de desmatamentos. As condições socioeconômicas e ambientais, associadas aos hábitos de vida, são importantes fatores na epidemiologia da LV em áreas endêmicas, favorecendo sua perpetuação nas áreas rurais e periurbanas, acometendo habitantes de aglomerados urbanos com baixo nível socioeconômico, cujas famílias vivem em condições precárias ${ }^{(2,11)}$.

Objetivou-se, neste estudo, verificar a prevalência da LVC em área periurbana com foco de transmissão (Condomínio Entrelagos - DF) no Distrito Federal e, também, avaliar a prevalência de coinfecções como dirofilariose, anaplasmose, erliquiose e doença de Lyme em cães sororreagentes para LVC.

\section{Material e métodos}

A região de escolha para o estudo foi uma área periurbana com foco de transmissão de leishmaniose visceral, onde foram confirmados dois casos de leishmaniose humana autóctone em $2015^{(12)}$. O Condomínio Entrelagos DF, situado na cidade-satélite de Sobradinho, no Distrito Federal, é delimitado pelas coordenadas $-15^{\circ} .7527438,-47^{\circ} .7536566$, e se encontra a $25 \mathrm{~km}$ de distância do Plano Piloto e a $22 \mathrm{~km}$ de distância da cidade de Sobradinho. O local conta com todas as ruas asfaltadas e se encontra próximo à estrada DF 250, estabelecendo limites com dois condomínios e o núcleo rural de Sobradinho. O Condomínio Entrelagos é uma região periurbana no Distrito Federal que possui 354 hectares e ocupa a área de uma antiga fazenda chamada Paranoá, localizada em região de alta altitude (entre 1138 e 1150 metros acima do nível do mar). Contida nesse condomínio, há uma reserva ecológica de 50 hectares que margeia o córrego Indaiá e abriga exemplares típicos da flora e da fauna da região do Cerrado. A região é dividida em quatro etapas, sendo que os lotes habitacionais têm no mínimo $1000 \mathrm{~m}^{2}$ e alta densidade populacional de cães, domiciliados ou não. A coleta de lixo é realizada duas vezes por semana.

Todos os 240 cães (Canis familiaris - LINNAEUS, 1758) domiciliados do Condomínio Entrelagos (DF) foram submetidos à coleta de sangue executada pelos agentes de saúde da Diretoria de Vigilância Ambiental em Saúde da Secretaria de Saúde do Distrito Federal, no período compreendido entre janeiro e dezembro de 2016. Previamente às coletas de sangue, foram obtidas informações com dados do tutor (nome, endereço) e do cão (raça, idade, gênero, entre outros).

As amostras de 3 a $5 \mathrm{~mL}$ de sangue foram obtidas da veia jugular, utilizando-se agulhas $25 \times 7 \mathrm{~mm}$ e tubos Vacutainer ${ }^{\circledR}$ sem anticoagulante. Posteriormente, as amostras foram centrifugadas a $800 \mathrm{G}$ durante cinco minutos para obtenção de amostras de soro, que foram armazenadas em temperatura de $-20^{\circ} \mathrm{C}$ até o momento das análises.

As amostras armazenadas foram utilizadas para a realização de exames diagnósticos para LVC, sendo inicialmente avaliadas pelo teste rápido de triagem, de maior sensibilidade, isto é, a reação imunocromatográfica que contém dois antígenos recombinantes - rK39 e rK26 - (DPP 
- LVC-Bio-Manguinhos - FIOCRUZ - Rio de Janeiro - RJ) para Leishmania sp. As amostras de soro dos cães reagentes ao teste de DPP foram reprocessadas e reavaliadas para LVC com ELISA, visando à confirmação do diagnóstico (maior especificidade). Mediante confirmação diagnóstica a ambos os testes, realizou-se avaliação sorológica para outras doenças infecciosas através do teste de SNAP (IDEXX 4Dx Plus), como Dirofilariose [D. immitis], Anaplasmose [Anaplasma phagocytophilum ou Anaplasma platys], Erliquiose [Ehrlichia canis ou Ehrlichia ewangii] e Doença de Lyme [Borrelia burgdorferi]) para se descartar coinfecções que potencialmente interferem no diagnóstico sorológico (falso-positivos).

Este estudo foi submetido e aprovado pelo Comitê de Ética de Uso Animal da Universidade de Brasília (CEUA-UnB), n. 31897/2016.

\section{Resultados e discussão}

Foram testados 240 cães domiciliados no Condomínio Entrelagos (Sobradinho-DF) entre janeiro e dezembro de 2016, sendo que $132(55 \%$ - 132/240) cães obtiveram resultados reagentes para DPP e para ELISA e, portanto, com diagnóstico positivo para LVC. A alta soroprevalência desta região se aproxima à demonstrada em estudos semelhantes, que chegaram a 75,3\% de positividade em cães em Anastásio (MS), ${ }^{(13)}$ enquanto outros estudos demonstraram prevalências entre $21 \%$ a $25 \%$ em cães de Jequié - BA ${ }^{(14)}$ e de São José do Ribamar - $\mathrm{MA}^{(15)}$.

Nas 132 amostras soropositivas para LVC, foram realizados testes para outras doenças infecciosas como dirofilariose, anaplasmose, erliquiose e doença de Lyme (SNAP- IDEXX 4Dx Plus) para se descartarem coinfecções que potencialmente interferem no diagnóstico sorológico (falso-positivos). Destas, 26 amostras (20\% - 26/132) foram reagentes para Ehrlichia canis ou Ehrlichia ewangii, prevalência próxima ao relatado em um estudo no estado do Paraná, que verificou coinfecção em $23 \%$ dos cães com LV ${ }^{(16)}$. Um total de nove amostras (7\% - 9/132) apresentou positividade para Anaplasma phagocytophilum ou Anaplasma platys. Coinfecção concomitante com anaplasmose, erliquiose e leishmaniose foi observada em 3 dos 132 (2,3\% - 3/132) ensaios realizados.

Embora o método de maior especificidade para o diagnóstico de LV seja o exame parasitológico, nem sempre ele é de fácil execução, e sua sensibilidade é relativamente baixa. Portanto, a incorporação de um teste rápido (DPP) no protocolo atual do PCVLV para detecção de LVC acelerou a implementação das medidas de controle em áreas endêmicas. O DPP é um procedimento que não requer equipamentos e suprimentos especializados e utiliza pequenas amostras de sangue. Já do ponto de vista da saúde pública, os resultados positivos em testes sorológicos são utilizados como critério, de acordo com o PCVLV, para indicação de eutanásia em cães reagentes ${ }^{(2,17)}$.

No entanto, esses testes sorológicos apresentam limitações importantes, como reações cruzadas - que permanecem controversas - com parasitas Trypanosoma, espécies de leishmaniose cutânea e outros hemoparasitas (Anaplasma spp., Ehrlichia canis, Babesia canis, Toxoplasma gondii e Neospora caninum), bem como resultados falso-negativos em casos de anergia ou baixos títulos (reações duvidosas) ${ }^{(18)}$. Ainda, estudos com o uso do método molecular (PCR) para detecção de Leishmania chagasi demonstraram ser essenciais para o diagnóstico acurado da LVC, especialmente em cães assintomáticos provenientes de áreas não endêmicas, nas quais os testes sorológicos não conseguiram acusar um número expressivo de animais infectados $(19,6 \% \text { - falso-negativos })^{(17,19)}$

Durante a vida, qualquer ser vivo é exposto a uma diversidade de parasitos de modo sequencial ou 
simultâneo, sendo rara a situação na qual há somente um parasita atuando num mesmo organismo hospedeiro. Provavelmente coexistem infecções de diferentes espécies de parasitas, ou de grupos taxonômicos ou gêneros diferentes ${ }^{(20,21)} \mathrm{e}$, dependendo da associação desses organismos, é notável sua competência em suprimir e/ou desviar a resposta imune do hospedeiro, com mecanismos tão diversos quanto os nichos que ocupam ${ }^{(22)}$. As associações entre parasitos são complexas e dependem de variáveis pertencentes ao meio e ao hospedeiro, gerando diversas manifestações clínicas que podem imunossuprimir o hospedeiro e induzir à má nutrição, considerada a causa mais importante de imunodeficiência secundária e de alta prevalência de infecções e doenças ${ }^{(23)}$.

A infecção dos cães pela Leishmania chagasi, Anaplasma platys e Ehrlichia canis coexistem devido ao fato de a atividade de seus vetores e o período de transmissão serem semelhantes, como também pela abundância de artrópodes vetores e de cães nas áreas endêmicas ${ }^{(24,25,26,27)}$. A ocorrência das espécies de Leishmania sp. é normalmente limitada à distribuição do vetor, que é determinada geograficamente. Os vetores pertencem à subfamília Phlebotominae, que se divide em dois gêneros principais: Phlebotomus e Lutzomyia. Na Europa, na Ásia e na África (Velho Mundo), os vetores das espécies causadoras das leishmanioses visceral e cutânea são do gênero Phlebotomus ${ }^{(28)}$ e, no Brasil, são do gênero Lutzomyia ${ }^{(2)}$.

A mudança no contexto da doença, que tem se tornado cada vez mais cosmopolita, é fato bastante conhecido. A devastação de grandes áreas silvestres para exploração econômica traz a doença para a periferia dos centros urbanos, sendo que tanto os vetores como os hospedeiros, nos casos silvestres, são obrigados a migrarem para o peridomicílio humano em busca de alimentos, transmitindo ao mesmo tempo os agentes da doença. A presença de animais domésticos e silvestres no peridomicílio atrai um grande número de flebotomíneos, aumentando o risco de transmissão da Leishmania sp. $^{(29)}$.

O Condomínio Entrelagos é uma região periurbana no Distrito Federal, no qual se encontra uma reserva ecológica constituída por exemplares da fauna do Cerrado e alta densidade populacional de cães com e sem tutores, justificando a alta incidência de animais soropositivos para LVC. A doença é caracterizada como endêmica em regiões periurbanas associadas ao desmatamento, acometendo cães e animais silvestres como reservatórios, principalmente as raposas (Dusicyon vetulus), os cachorrosdo-mato (Cerdocyon thous) e os marsupiais (gambás - Didelphis albiventris) ${ }^{(30)}$. A LVC é uma doença sistêmica severa cujas manifestações clínicas são intrinsecamente dependentes do tipo de resposta imunológica expressa pelo animal infectado, sendo que o próprio parasito é capaz de causar e perpetuar essa imunossupressão permitindo sua disseminação para muitos órgãos, com agravamento do quadro clínico do paciente ${ }^{(2,18,26,27)}$.

Os cães são considerados os principais reservatórios do protozoário no âmbito doméstico devido ao elevado parasitismo cutâneo que esse animal consegue apresentar, facilitando a contaminação do vetor, que já tem natural atração para esse vertebrado de sangue quente ${ }^{(31)}$. A justificativa da erradicação do cão positivo para LVC baseia-se, principalmente, no contato íntimo do cão com o ser humano, já que casos de LVC antecedem os casos em seres humanos nas áreas urbanas do Brasil ${ }^{(32)}$ e, mesmo apesar de a frequência de infecção de ambos apresentar diferenças, o parasita dos dois hospedeiros é o mesmo. Entretanto, a eutanásia de animais positivos é vista como equivocada por muitos estudiosos para o combate à disseminação da doença, pois, além de apresentar diagnósticos que não são suficientemente acurados para essa conduta ${ }^{(10,18,19)}$, principalmente pela baixa sensibilidade dos métodos sorológicos utilizados ${ }^{(7,18,19)}$, a literatura reporta o pouco impacto dessa prática sobre a redução de casos humanos e caninos ${ }^{(33,34,35)}$. 


\section{Conclusão}

No presente estudo, foi observada importante prevalência de animais sororreagentes para LVC em região periurbana do Distrito Federal, muitos dos quais cursando com coinfecções concomitantes, com destaque para a erliquiose. Verifica-se a necessidade de diagnóstico mais preciso quanto a coinfecções com a LVC que podem influenciar no prognóstico e no quadro clínico desses pacientes.

\section{Agradecimentos}

À Diretoria de Vigilância Ambiental em Saúde (DIVAL/SES/DF) do Distrito Federal e ao CNPq, edital PIBIC.

\section{Referências}

1. Feitosa MM, Ikeda FA, Luvizotto MCR, Perri SHV. Aspectos clínicos de cães com leishmaniose visceral no município de Araçatuba - São Paulo (Brasil). Clínica Veterinária. 2000, 15(28):36-44.

2. Brasil, Ministério da Saúde. Secretaria de Vigilância em Saúde. Depto. de Vigilância Epidemiológica. Manual de vigilância e controle da leishmaniose visceral, 2014. Disponí-vel em: http://bvsms.saude.gov.br/ bvs/publicacoes/manual_vigilancia controle leishmaniose visceral 1edicao.pdf

3. Alvar J, Canavate C, Molina R, Moreno J, Nieto J. Canine leishmaniasis. Advances in Parasitology. 2004, 57: 1-88. Disponível em: https://doi.org/10.1016/S0065-308X(04)57001-X

4. Molina R, Amela C, Nieto J, Sandres M, Gonzalez F, Castillo JA, et al. Infectivity of dogs naturally infected with Leishmania infantum to colonized Phlebotomus perniciosus. Transactions of the Royal Society of Tropical Medicine and Hygiene. 1994, 88: 491-493. Disponível em: https://doi.org/10.1016/0035-9203(94)90446-4.

5. Guiunchetti RC, Mayrink W, Genaro O, Carneiro CM, Corrêa-Oliveira R, Martins-Filho OA, et al. Relationship between canine visceral leishmaniosis and the Leishmania (Leishmania) chagasi burden in dermal inflammatory foci. Journal of Comparative Pathology. 2006,135:100-107. Disponível em: https://doi. org/10.1016/j.jcpa.2006.06.005

6. Michalsky EM, Rocha MF, Da Rocha Lima AC, França-Silva JC, Pires MQ, Oliveira FS, et al. Infectivity of seropositive dogs, showing different clinical forms of leishmaniasis, to Lutzomyia longipalpis phlebotomine sand flies. Veterinary Parasitology. 2007,147: 67-76. Disponível em: https://doi.org/10.1016/j.vetpar.2007.03.004

7. Romero GAS, Boelaert M. Control of visceral leishmaniasis in Latin America-A Systematic Review. PLoS Neglected Tropical Disease. 2010, 4(1):e584. Disponível em: https://doi.org/10.1371/journal.pntd.0000584

8. Brasil. Ministério da Saúde. Esclarecimento sobre substituição do protocolo diagnóstico da leishmaniose visceral canina; Nota técnica conjunta nu 01/2011 - CGDT-CGLAB/DEVIT/SVS/MS. 2011.

9. Coura-Vital W, Ker HG, Roatt BM, Aguiar-Soares RD, Leal GG, Moreira ND, et al. Evaluation of change in canine diagnosis protocol adopted by the Visceral Leishmaniasis Control Program in Brazil and a new proposal for diagnosis. PLoS ONE. 2014, 9(3): e91009. Disponível em: https://doi:10.1371/journal.pone.0091009

10. Faria AR, De Andrade HM. Diagnóstico da Leishmaniose Visceral Canina: grandes avanços tecnológicos e baixa aplicação pratica; Rev Pan-Amaz Saúde 2012. p.47. Disponível em: http://dx.doi.org/10.5123/S217662232012000200007 
11. Coutinho MT, Bueno LL, Sterzik A, Fujiwara RT, Botelho JR, De Maria M, et al. Participation of Rhipicephalus sanguineus (Acari: Ixodidae) in the epidemiology of canine visceral leishmaniasis. Veterinary Parasitology. 2005, 128(1-2):149-155. Disponível em: https://doi.org/10.1016/j.vetpar.2004.11.011

12. Secretaria de Estado de Saúde. Informativo Epidemiológico das Leishmanioses no Distrito Federal. Gerência de Doenças Crônicas e Outros Agravos Transmissíveis (GEDCAT). Subsecretaria de vigilância à saúde. Secretaria de Estado de Saúde. Governo do Distrito Federal. 2016. 8p. Disponível em: http://www.saude. df.gov.br/images/Informativos/2016/Informativo Epidemiologico das Leishmanioses no DF 1 2016.pdf

13. Cortada VMCL, Doval MEC, Souza-Lima MAA, Oshiro ET, Neneses CRV, Abreu-Silva AL, et al. Canine visceral leishmaniasis in Anastácio, Mato Grosso do Sul State, Brazil. Veterinary Research Communications. 2004, 28(5): 365-374. Disponível em: https://doi.org/10.1023/B:VERC.0000035014.80785.b7

14. Paranhos-Silva M, Freitas LAR, Santos WC, Grimaldi GJ, Pontes-de-Carvalho LC, Oliveira-dos-Santos AJ. A cross-sectional serodiagnostic survey of canine leishmaniasis due to Leishmania chagasi. American Journal of Tropical Medicine Hygiene. 1996, 55(1): 39-44. Disponível em: https://doi.org/10.4269/ajtmh.1996.55.39

15. Guimarães KS, Batista ZS, Dias EL, Guerra RMSNC, Costa ADC, Oliveira AS, et al. Canine visceral leishmaniasis in São José de Ribamar, Maranhão State, Brazil. Veterinary Parasitology. 2005, 131(3-4): 305309. Disponível em: https://doi.org/10.1016/j.vetpar.2005.05.008

16. Trapp SM, Dagnone AS, Vidotto O, Freire RL, Morais HSA. Seroepidemiology of ca-nine babesiosis and ehrlichiosis in a hospital population. Veterinary Parasitology. 2006, 140(3-4): 223-230. Disponível em: https:// doi.org/10.1016/j.vetpar.2006.03.030

17. Lopes EG, Sevá AP, Ferreira F, Nunes CM, Keid LB, Hiramoto RM, et al. Serological and molecular diagnostic tests for canine visceral leishmaniasis in Brazilian endemic area: one out of five seronegative dogs are infected. Epidemiology \& Infection. 2017, 145(12):2436-2444. Disponível em: https://doi.org/10.1017/ $\underline{\text { S0950268817001443 }}$

18. Zanette MF, Lima VMF, Laurenti MD, Rossi CN, Vides JP, Vieira RFC, et al. Serological cross-reactivity of Trypanosoma cruzi, Ehrlichia canis, Toxoplasma gondii, Neospora caninum and Babesia canis to Leishmania infantum chagasi tests in dogs. Revista da Sociedade Brasileira de Medicina Tropical. 2014, 47(1). Disponível em:http://dx.doi.org/10.1590/0037-8682-1723-2013

19. Riboldi E, Carvalho F, Roosevelt P, Romão T, Barcellos RB, Bello GL, et al. Molecular method confirms canine leishmania infection detected by serological methods in non-endemic area of Brazil. Korean J Parasitol. 2018, 56(1):11-19. Disponível em: https://doi.org/10.3347/kjp.2018.56.1.11

20. Cox FEG. Concomitant infections, parasites and immune responses. Parasitology. 2001,122: S23-38. Disponível em: https://doi.org/10.1017/S003118200001698X

21. Mckay DM. The beneficial helminth parasite? Parasitology. 2006, 132:1-12. Disponível em: https://doi. org/10.1017/S003118200500884X

22. Pfaff AW, Candolfi E. Immune responses to protozoan parasites and its relevance to diagnosis in immunocompromised patients. European Journal of Protistology. 2003, 39: 428-34. Disponível em: https:// doi.org/10.1078/0932-4739-00016

23. Lloyd, S. (1995). Environmental Influences on Host Immunity. In B. Grenfell \& A. Dobson (Eds.), Ecology of Infectious Diseases in Natural Populations (Publications of the Newton Institute, pp. 327-361). Cambridge: Cambridge University Press. Chapter DOI: https://doi.org/10.1017/CBO9780511629396.013

24. Grooves MG, Dennis GL, Amyx HL, Huxsoll DL. Transmission of Ehrlichia canis to dogs by ticks (Rhipicephalus sanguineus). American Journal of Veterinary Research, 1975, 36(7): 937-940. 
25. Inokuma H, Raoult D, Brouqui P. Detection of Ehrlichia platys DNA in brown dog ticks (Rhipicephalus sanguineus) in Okinawa Island, Japan. Journal of Clinical Microbiol-ogy. 2000, 38(11): 4219-4221. Disponível em: http://jem.asm.org/content/38/11/4219.full

26. Mekuzas Y, Gradoni L, Oliva G, Foglia Manzillo V, Baneth G. Ehrlichia canis and Leishmania infantum co-infection: a 3-year longitudinal study in naturally exposed dogs. Journal Compilation 2009 European Society of Clinical Microbiology and Infectious Diseases, 15 (Suppl. 2):30-31. Disponível em: http://dx.doi. org/10.1111/j.1469-0691.2008.02150.x

27. Souza KCM. Co-infecções por Ehrlichia canis, Leishmania chagasi e Babesia canis em cães naturalmente infectados em Campo Grande, Mato Grosso do Sul. Jaboticabal (SP): Universidade Estadual Paulista "Júlio de Mesquita Filho"; 2012. Disponível em: http://200.145.6.238/bitstream/handle/11449/96002/sousa kcm me jabo.pdf? sequence $=1$ \&isAllowed $=\mathrm{y}$

28. Greene C. Ehrlichiosis, Neorickettsiosis, Anaplasmosis and Wolbachia Infection. In: Greene C. Infectious diseases of the dog and cat. 3rd ed. St. Louis: Saunders; 2006. p. 203 -231.

29. Barata AR, França-Silva CJ, Mayrink W, Da Silva CJ, Prata A, Loroza SE, et al. Aspectos da ecologia e do comportamento de flebotomíneos em área endêmica de leishmaniose visceral, Minas Gerais. Revista da Sociedade Brasileira de Medicina Tropical. 2005, 38(5):421-425. Disponível em: http://dx.doi.org/10.1590/ S0037-86822005000500012

30. Santiago MEB, Vasconcelos RO, Fattori KR, Munari DP, Michelin AF, Lima VMF. An investigation of Leishmania spp. in Didelphis spp. from urban and periurban areas in Bauru (Sao Paulo, Brazil). Veterinary Parasitology. 2007, 150: 283-290. Disponível em: https://doi.org/10.1016/j.vetpar.2007.09.026

31. Mathis A, Deplazes P. PCR and in vitro cultivation for detection of Leishmania spp. in diagnostic samples from humans and dogs. Journal of Clinical Microbiology. 1995, 33(5)1145-1149. Disponível em: https://www. ncbi.nlm.nih.gov/pmc/articles/PMC228120/pdf/331145.pdf

32. Herenio ME, Fortes RC, Rincon G. Prevalência da Leishmaniose visceral em cães do Distrito Federal, segundo dados do centro de zoonoses de Brasília. J Health Sci Inst. 2014. 32(2):126-9. Disponível em: https:// www.unip.br/comunicacao/publicacoes/ics/edicoes/2014/02_abr-jun/V32_n2_2014_p126a129.pdf

33. Courtenay O, Quinnel RJ, Garcez LM, Shaw JJ, Dye C. Infectiousness in a cohort of brazilian dogs: why culling fails to control visceral leishmaniasis in areas of high transmission. Journal of Infectious Disease. 2002, 186 (9): 1314-1320. Disponível em: https://doi.org/10.1086/344312

34. Costa Ch. How effective is dog culling in controlling zoonotic visceral leishmaniasis? A critical evaluation of the science, politics and ethics behind this public health policy. Revista da Sociedade Brasileira de Medicina Tropical. 2011, 44(2):232-242. Disponível em: http://dx.doi.org/10.1590/S0037-86822011005000014

35. Grimaldi G, JR., Teva A, Santos CB, Ferreira AL, Falqueto A. The effect of removing potentially infectious dogs on the numbers of canine Leishmania infantum infections in an endemic area with high transmission rates. The American Journal of Tropical Medicine and Hygiene. 2012, 86(6): 966-971. Disponível em: https:// doi.org/10.4269/ajtmh.2012.12-0040 